\title{
Use of oil-rich diet for gaited horses during physical training
}

\author{
Hélio Cordeiro Manso Filho, Monica Miranda Hunka, Luzilene Araújo de Souza, \\ Helena Emília Cavalcanti da Costa Cordeiro Manso
}

\author{
Federal Rural University of Pernambuco, Equine Research Center, Recife, PE, Brazil
}

Received August 2, 2018

Accepted February 12, 2019

\begin{abstract}
In Brazil, gaited horses are selected based on field tests, during which they move at speeds of 3 to $4 \mathrm{~m} / \mathrm{s}$ for 30 to $60 \mathrm{~min}$. To cover their nutrient requirements, feed manufacturers have developed oil-rich dietary supplements and concentrates. The aim of this research was to evaluate the effects of increasing the density of dietary fats in the feed of 16 Campolina horses undergoing intense gaited training. The training schedule consisted of training $4 \times$ a week: 3 days riding for 60 min (10' warm-up, 40' doing marcha gait, and 10' cool down, and 1 day walking for 90'). The horses were divided in two groups: control and supplemented. The dietary supplementation was isocaloric, with the control group receiving an ordinary concentrate $(3.5 \%$ fat $)$ and the supplemented group fed $1.0 \mathrm{~kg}$ of the supplement $(18 \%$ fat $)$ plus the ordinary concentrate. Both groups had free access to fresh grass, salt and water. Blood samples were collected prior to and after 4 and 8 weeks of supplementation to calculate the complete blood count, glucose, total protein, triglycerides, total cholesterol, HDL, LDL and non-esterified fatty acids (NEFA). The results were evaluated by ANOVA and Tukey's test $(P<0.05)$. The supplemented group showed elevated levels of NEFA, red blood cells and haematocrit $(P<0.05)$, while the control group showed high triglyceride concentrations; both groups showed reduced plasma protein concentrations $(P<0.05)$. The other indices did not change $(P>0.05)$. The increase in dietary fat intake increased the blood lipid biomarkers and antioxidant capacity of gaited horses during intense training, possibly contributing to improve their metabolic performance.
\end{abstract}

Aerobic exercise, antioxidant, cholesterol, NEFA, triglycerides

Over the last few decades, the increasing popularity of equestrian sports such as endurance and pacer riding events has led to changes in dietary standards for these athletes, in terms of both energy sources and the increase in sources of antioxidant nutrients. Thus, the use of concentrates of high calorie density due to the addition of oils allows for the inclusion of nutrients to cover the energy expenditure during training and competitions, improving metabolic efficiency for endurance training by reducing muscle glycogen depletion and improving the stamina of these athletes (Kronfeld et al. 1994; Harris 2009). However, other studies have found that the increase in ethereal extract in diets can reduce the elevation of body temperature and water needs induced by exercise (Kronfeld et al. 1994; Hyyppa et al. 1999). These facts are important and should be kept in mind when the inclusion of dietary oils is prescribed for speed and endurance exercises because, in the long run, they contribute to the longevity and well-being of equine athletes.

It has also been shown that the inclusion of oils rich in omega 3 and 6 fatty acids could contribute to improve antioxidant capacity of different groups of horse athletes. It has been shown that the tissues of endurance riding and gaited horses undergo a major loss of antioxidant capacity during competition (Hargreaves et al. 2002; Melo et al. 2017). That is why the use of antioxidant-rich dietary oils may contribute to improve the performance of endurance athletes. It has recently been shown that the inclusion of oils rich in omega 3 and 6 fatty acids in the diet of horses undergoing 8 weeks of gait training increased the concentration of superoxide dismutase (SOD), glutathione peroxidase (GPx) and uric acid

Address for correspondence:

Hélio Cordeiro Manso Filho

Núcleo de Pesquisa Equina

Universidade Federal Rural de Pernambuco

Rua Dom Manuel de Medeiros s/n, 52171-900 Recife, PE, Brasil.

E-mail: helio.mansofo@ufrpe.br

http://actavet.vfu.cz/ 
(Melo et al. 2016). In weaned foals, an increase in the percentage of the ethereal extract in diet reduced the degree of anisocytosis and increased the leukocyte concentration. This effect was attributed to the improved antioxidant functions in these cells, followed by the positive effects on plasma cell membrane stability (Moffarts et al. 2007; Melo et al. 2012).

However, it should be kept in mind that a calorie increment through the inclusion of oils in food concentrates can lead to reduced food intake, faecal production and plasma triglyceride concentration, and can extend the recovery time of muscle glycogen concentrations (Kronfeld et al. 1994; Orme et al. 1997; Hyyppa et al. 1999; O'Connor et al. 2007). Finally, it should be noted that the time required for the body's metabolism to fully adapt to the use of dietary oil supplementation may vary according to the type and amount of oil used, and that many of these effects quickly disappear when the supplementation is discontinued (Hyyppa et al. 1999; O’Connor et al. 2007; NRC 2007).

Campolina horses are a typical Brazilian breed. They are able to perform a four-beat gait exercise used in marcha challenges that consist of an aerobic exercise. Given the importance of including oil in the diet of equine athletes, not only as a source of energy but also to improve the animal's antioxidant capacity, an experiment was performed to determine the effects of an increased ethereal extract in the diet of horses undergoing gait training on their body condition score, lipid biomarkers, and complete blood count (CBC). Maintaining the body condition score and increasing the concentrations of free fatty acids (FFAs) in the blood of horses undergoing endurance training may improve their performance due to the greater contribution of calories typically used in these sports, and may also improve the antioxidant capacity and maintain the composition of the plasma membrane.

The aim of this research was to determine the effects of oil supplementation on blood biomarkers in gaited horses under intense training program.

\section{Materials and Methods}

This study involved 16 Campolina horses, which were randomly assigned to two groups - a supplemented group and a control group, each comprising 4 males and 4 non-pregnant females. The horses were of the mean age of 6.5 years and body weight of $480 \mathrm{~kg}$. All the horses were housed in single stalls, but in visual contact with each other, and on their day off were allowed to graze on a 5-ha pasture covered with Massai grass (Panicum maximum). All the handling and training procedures at the experimental site involved the use of positive stimuli and reduction of negative stimuli, according to the five domains described in the literature (Mellor 2017; McGreevy et al. 2018). An evaluation scale of good practices for horse athletes was adopted for the training evaluation (Coelho et al. 2018), and all the practices, as well as sampling, were approved by the Ethics Committee on Animal Use of the Federal Rural University of Pernambuco - CEUA/UFRPE, under Protocol No. 026/2013.

Both groups spent more than 3 months by the training for gaited competitions. The training protocol consisted of 3 days/week riding for gaited competition training (10 min walking to warm up, followed by 40 min riding at pacer gait (3-4 m/s), and ending with 10 min walking to cool down), and 1 day/week walking outdoors for $90 \mathrm{~min}$. The rest of the time they stayed in their individual stalls $\left(16 \mathrm{~m}^{2}\right)$ at rest, or were allowed to graze freely for a maximum of $5 \mathrm{~h}$ per day on the pasture.

The animals were fed isocaloric diets in individual troughs, $3 \times$ a day. The control group received only a commercial concentrate $(5.0 \mathrm{~kg}$ /day per animal, CP [crude protein]: $12 \%$, EE [ether extract]: $3.5 \%, \mathrm{DE}$ [digestible energy]: $3.0 \mathrm{Mcal} / \mathrm{kg})$, while the supplemented group was fed the commercial concentrate $(3.5 \mathrm{~kg} / \mathrm{day}$ per animal) plus an oil-rich supplement (1.0 kg/animal/day, CP: $10.0 \%$, EE: $18.0 \%$, DE: 4.4Mcal). In addition to the concentrates, all the animals fed on fresh Massai grass, about $15 \mathrm{~kg} / \mathrm{animal} / \mathrm{day}$, and had ad libitum access to salt and water. The body condition score was determined on a scale of 1 to 9 at three time points: before, 30 days, and 60 days after starting supplementation, as described in the literature (Henneke et al. 1983). The recovery heart rate was measured once a week, using a stethoscope applied to the left side of the heart region, 20 to 30 min after the gait training exercises.

Blood samples were collected before beginning the supplementation, and 4 weeks and 8 weeks after the supplementation. All samples were drawn from fasting animals by jugular venipuncture into heparin vacuum tubes. The whole blood was used to perform blood cell counts within no more than $2 \mathrm{~h}$ after collection. The samples were then centrifuged to separate the plasma, which was used for the determination of glucose, total plasma protein (TPP), FFAs, triglycerides, total cholesterol, high-density lipoprotein (HLD) cholesterol and low-density lipoprotein (LDL) cholesterol. The complete blood count (CBC) was performed in semi- 
automated haematology analyser (Sysmex pocH-100iV Diff, Roche, São Paulo, Brazil), and biochemical analyses were carried out in a semi-automated spectrophotometer (Doles D-250, Goiás, Brazil) using commercial kits.

One-way ANOVA (treatment) and Tukey's test were employed to analyse the results. In both cases, the $P$ value was set at 0.05. The analyses were performed using SigmaPlot 13.0 for Windows (Systat Software Inc., San Jose, CA, USA). The results are expressed as mean \pm standard error.

\section{Results}

The results indicated that, at the end of the 8 weeks, the non-esterified fatty acid (NEFA) concentrations in the supplemented group were 10-fold higher than those before the testing and in the control group $(P<0.05)$. The control group showed a higher triglyceride concentration before the testing and over the time than the supplemented group $(P<0.05)$ (Table 1). Total plasma protein (TPP) contents were also found to decrease over the time in both groups compared to the level before the testing $(P<0.05)$. However, the body condition score, glucose concentration and cholesterol (total, LDL and HDL) did not vary throughout the experiment $(P>0.05)$.

Table 1. Body condition score and chemical biomarkers of athletic gaited horses without and with supplementation of concentrate rich in ethereal extract.

\begin{tabular}{lccccc}
\hline & & \multicolumn{3}{c}{ Group of athletic horses } \\
\cline { 3 - 6 } Biomarker & Before testing & \multicolumn{2}{c}{ Control $(\mathrm{n}=8)$} & \multicolumn{2}{c}{ Supplemented $(\mathrm{n}=8)$} \\
\cline { 3 - 6 } & $(\mathrm{n}=16)$ & $+30 \mathrm{~d}$ & $+60 \mathrm{~d}$ & $+30 \mathrm{~d}$ & $+60 \mathrm{~d}$ \\
\hline Body condition score & $5.2 \pm 0.2$ & $5.3 \pm 0.2$ & $5.9 \pm 0.2$ & $5.0 \pm 0.2$ & $5.9 \pm 0.1$ \\
Glucose, mmol/1 & $4.92 \pm 0.11$ & $5.24 \pm 0.01$ & $5.06 \pm 0.07$ & $5.11 \pm 0.05$ & $4.83 \pm 0.11$ \\
Total plasma protein, g/dl & $7.8 \pm 0.2^{\mathrm{A}}$ & $7.2 \pm 0.1^{\mathrm{B}}$ & $6.8 \pm 0.2^{\mathrm{B}}$ & $7.2 \pm 0.1^{\mathrm{B}}$ & $7.1 \pm 0.1^{\mathrm{B}}$ \\
NEFA, mmol/ml & $0.034 \pm 0.004^{\mathrm{B}}$ & $0.053 \pm 0.005^{\mathrm{B}}$ & $0.030 \pm 0.008^{\mathrm{B}}$ & $0.106 \pm 0.017^{\mathrm{AB}}$ & $0.361 \pm 0.167^{\mathrm{A}}$ \\
Triglycerides, mmol/1 & $0.33 \pm 0.02^{\mathrm{A}}$ & $0.31 \pm 0.02^{\mathrm{AB}}$ & $0.28 \pm 0.04^{\mathrm{AB}}$ & $0.22 \pm 0.04^{\mathrm{B}}$ & $0.19 \pm 0.02^{\mathrm{B}}$ \\
Total cholesterol, mmol/1 & $2.25 \pm 0.12$ & $2.17 \pm 0.08$ & $2.34 \pm 0.12$ & $2.50 \pm 0.15$ & $2.77 \pm 0.27$ \\
HLD cholesterol, mmol/1 & $1.17 \pm 0.06$ & $1.19 \pm 0.05$ & $1.46 \pm 0.29$ & $1.33 \pm 0.06$ & $1.28 \pm 0.09$ \\
LDL cholesterol, mmol/1 & $1.08 \pm 0.12$ & $0.97 \pm 0.07$ & $1.24 \pm 0.07$ & $1.17 \pm 0.13$ & $1.49 \pm 0.19$ \\
\hline
\end{tabular}

Different superscripts in one row indicate that $P<0.05$ by Tukey's test. NEFA - non esterified fatty acids.

The evaluation of the haematological biomarkers revealed that the erythrocyte counts and haematocrit levels increased in the supplemented group $(P<0.05)$, with the highest levels measured after 8 weeks of supplementation (Table 2). All the other evaluated indices remained unchanged over the 8 weeks of the experiment.

Lastly, it should be noted that during the experimental period, the animals consumed all the concentrated feed without any problem, and their clinical status remained unchanged. Moreover, the animals showed no lameness, injuries or other problems related to training during the experiment. The recovery heart rate was consistent with the welfare assessment scale for equine athletes (Coelho et al. 2018) on the days when the horses did their walking exercises.

\section{Discussion}

This study demonstrated that the inclusion of an oil-rich dietary concentrate over an 8-week period resulted in a significant increase in NEFA levels in the supplemented group, even when these horses underwent intense competitive training. This supplementation was 
Table 2. Blood biomarkers of athletic gaited horses without and with supplementation of concentrate rich in ethereal extract

\begin{tabular}{|c|c|c|c|c|c|}
\hline \multirow{3}{*}{ Biomarker } & \multirow{3}{*}{$\begin{array}{l}\text { Before testing } \\
\quad(\mathrm{n}=16)\end{array}$} & \multicolumn{4}{|c|}{ Group of athletic horses } \\
\hline & & \multicolumn{2}{|c|}{ Control $(\mathrm{n}=8)$} & \multicolumn{2}{|c|}{ Supplemented $(\mathrm{n}=8)$} \\
\hline & & $+30 \mathrm{~d}$ & $+60 d$ & $+30 \mathrm{~d}$ & $+60 d$ \\
\hline Erythrocyte count, $\mathrm{X} 10^{6} / \mu 1$ & $7.6 \pm 0.4^{\mathrm{B}}$ & $7.5 \pm 0.3^{\mathrm{B}}$ & $7.7 \pm 0.3^{\mathrm{AB}}$ & $8.8 \pm 0.3^{\mathrm{AB}}$ & $8.9 \pm 0.4^{\mathrm{A}}$ \\
\hline Haemoglobin, g/dl & $11.55 \pm 0.4$ & $11.9 \pm 0.6$ & $11.9 \pm 0.5$ & $12.8 \pm 0.4$ & $13.0 \pm 0.2$ \\
\hline Haematocrit, \% & $34.2 \pm 1.2^{\mathrm{B}}$ & $35.2 \pm 1.8^{\mathrm{AB}}$ & $35.9 \pm 1.2^{\mathrm{AB}}$ & $38.1 \pm 1.2^{\mathrm{AB}}$ & $39.6 \pm 0.9^{\mathrm{A}}$ \\
\hline $\mathrm{MCV}, \mathrm{fl}$ & $45.1 \pm 1.2$ & $46.9 \pm 1.7$ & $46.7 \pm 1.6$ & $43.5 \pm 1.2$ & $44.9 \pm 1.4$ \\
\hline $\mathrm{MCHC}, \mathrm{g} / \mathrm{dl}$ & $33.5 \pm 0.2$ & $33.8 \pm 0.3$ & $33.3 \pm 0.4$ & $33.7 \pm 0.3$ & $33.3 \pm 0.4$ \\
\hline RDW-SD, fl & $36.4 \pm 0.7$ & $37.9 \pm 0.9$ & $37.6 \pm 0.5$ & $36.5 \pm 0.5$ & $36.8 \pm 0.6$ \\
\hline RDW-CV, \% & $20.3 \pm 0.4$ & $20.0 \pm 0.5$ & $19.0 \pm 0.6$ & $21.0 \pm 0.7$ & $20.7 \pm 0.6$ \\
\hline Platelets, $\mathrm{X} 10^{3} / \mu \mathrm{l}$ & $134.5 \pm 18.1$ & $117.3 \pm 24.2$ & $110.7 \pm 10.2$ & $159.7 \pm 21.7$ & $160.0 \pm 19.8$ \\
\hline Leukocytes, $\mathrm{X} 10^{3} / \mu 1$ & $9.8 \pm 0.6$ & $9.2 \pm 0.8$ & $8.5 \pm 0.4$ & $9.8 \pm 0.7$ & $9.5 \pm 0.7$ \\
\hline Lymphocytes, $\mathrm{X} 10^{3} / \mu 1$ & $4.5 \pm 0.5$ & $4.3 \pm 0.6$ & $4.0 \pm 0.5$ & $5.3 \pm 0.6$ & $4.9 \pm 0.6$ \\
\hline Other cells, $X 10^{3} / \mu 1$ & $5.4 \pm 0.3$ & $4.8 \pm 0.4$ & $4.5 \pm 0.6$ & $4.5 \pm 0.2$ & $4.6 \pm 0.3$ \\
\hline
\end{tabular}

Different superscripts in one row indicate $P<0.05$ by Tukey's test. MCV - mean corpuscular volume; MCHC - mean corpuscular haemoglobin concentration; RDW-SD - red cell distribution width-standard deviation; RDW-CV - red cell distribution width-coefficient of variation

also found to elevate haematocrit levels and red blood cell counts, which are important factors for athletic performance, probably because they support the stabilization of red blood cell membranes (Moffarts et al. 2007). Both groups of horses also showed no modifications in their total cholesterol, HDL and LDL concentrations. Both groups showed low TPP levels throughout the experimental period. However, this may be attributed to the improved physical conditioning of the horses, revealed by regular heart rate monitoring, given that animals undergoing endurance training may have a high plasma volume (McKeever et al. 1987; McKeever 2002).

Although the assessment of the body condition score is a subjective measure, it can be an important tool for evaluating the nutritional and training program of animal athletes. Horses tend to lose or gain body mass when their feeding program and/or training intensity are not balanced. This indicator should also be used in equine welfare standards, since excesses are detrimental to the well-being of animals (Mellor 2017; Coelho et al. 2018). The body condition score not only enables the athlete's nutritional program to be evaluated, but also helps to clarify the effects of the training program. There is no specific body condition score for each equestrian discipline, but in general, racehorses have a lower body condition score and body fat index because their fat mass percentage is lower than that of gaited horses (Kearns et al. 2002; Abreu et al. 2009). Moreover, it should be kept in mind that the source of energy for racehorses is more closely associated with carbohydrates, whereas that of endurance horses is associated with oils.

In this experiment, modifications in the body condition score were not expected because the animals were receiving dietary supplementation for low to moderate intensity sports of medium to long-term duration, as recommended in the literature (NRC 2007), and because they were being properly trained for the type of exercise they engaged in and enjoyed regular rest. Maintenance of the body condition score indicates that good breeding and training practices were followed.

Supplementation with different types of fat has proved to be an important factor for improving the performance of animals during medium to long duration exercises. The NEFA and triglyceride levels before the testing were similar to those described for fasting 
Marchador horses (NEFA: $\sim 0.025 \mathrm{mmol} / \mathrm{ml}$; triglycerides: $\sim 32.0 \mathrm{mg} / \mathrm{dl}$ ) (Ferreira et al. 2017). However, in the supplemented group, NEFA levels increased while triglyceride levels decreased compared to the concentrations measured before the testing or in the control group, in addition to those described in the literature (Ferreira et al. 2015). The results of this experiment are similar to others reported in the literature, which indicate a reduction in triglyceride levels and an increase in NEFA levels, but differ in terms of total cholesterol (Gleenen et al. 1999). However, some differences may be attributed to the composition of the oil used in this study and the characteristics of the exercises that the animals performed.

The effects of exercising on lipid biomarkers may vary depending on the dietary oil supplementation and types of exercises. In racehorses, supplementation with oil elevated plasma lipase activity and muscle antioxidant capacity, but did not increase muscle glycogen and triglyceride levels (Orme et al. 1997). In contrast, gaited horses show increased NEFA and triglyceride levels during and immediately after exercise (Ferreira et al. 2015), similar to what has been described for other equine sport disciplines (Kronfeld 1996; Orme et al. 1997). In the latter disciplines, which are typically aerobic, the increased availability of fats in feed can support athletic performance because they provide high-energy sources and antioxidant capacity in the blood of horse athletes.

It is noteworthy that no changes were detected in the glucose or cholesterol (total, HDL and LDL) levels in either of the groups, unlike what has been described in the literature (Gleenen et al. 1999). With regard to blood glucose, this indicator was measured when the horses were fasting and no changes were expected, although it has been reported that the inclusion of dietary oil could modify the postprandial concentration of this metabolite (Orme et al. 1997). The availability of fat in the diet as an energy source of this group of horses could support lipolysis in the aerobic exercise and thus help maintain the glucose levels. Other authors have described changes in the different types of cholesterol, which in some cases have been ascribed to the type of fat used in supplementation (Orme et al. 1997; O'Connor et al. 2007). Thus, the kind of fat source used in the current study could explain the absence of changes in the cholesterol levels in the four-beat gait horses.

Dietary fat supplementation and its effects on equine erythrocytes and leukocytes have not yet been clearly described in the literature. The results of this experiment indicated that erythrocyte counts in the non-supplemented group, both before and throughout the experiment, were below the previously described levels (Ferreira et al. 2015). However, the dietary supplementation raised the erythrocyte counts to levels similar to those reported in the literature for Campolina and Mangalarga Marchador horses (Ferreira et al. 2015), while the leukocyte levels in all the animals of this experiment were within the range described by those authors.

Very few studies so far have attempted to determine the effects of the inclusion of dietary oil on the blood indices of horses. In young animals ( $<12$ months old), the inclusion of dietary oil was shown to lower one of the indices of anisocytosis and raise the percentage of lymphocytes (Melo et al. 2012), but in adult racehorses this inclusion led only to a discrete elevation in leukocytes and had no influence on erythrocytes (Harris et al. 1999). It has also been shown that increasing the amount of oil added to horse diets increases the concentration of blood antioxidant biomarkers (Melo et al. 2016), which may improve the stability of plasma membranes in blood cells (Moffarts et al. 2007), even when horse athletes are subjected to oxidative conditions, such as physical exercise. Thus, supplementation with oils may have indirect effects on performance by improving the haematological biomarkers.

Plasma membrane flexibility is maintained by achieving a better balance between oxidants and antioxidants, and is important for cell function (Hollán 1996; Moffarts et al. 2007). In this context, the inclusion of dietary oil may support a higher concentration 
of omega 3 and 6 fatty acids in the membranes of erythrocytes and leukocytes, thus stabilizing the plasma cell membranes and offsetting the antioxidant effects caused by different sources (Barros et al. 2013; Eltweri et al. 2017). The stabilization of these cell membranes supports not only oxygen transport through the erythrocytes but may also contribute to the immune system by stabilizing the leukocyte cell membranes. Marchador horses are known to be subject to lymphopaenia and reduced antioxidant capacity following marcha contests (Wanderley et al. 2015; Melo et al. 2017), similar to what occurs in endurance riding horses or in other endurance contests. Thus, the increment in calories and antioxidants afforded by supplementation with oil rich in omega 3 and 6 fatty acids may contribute to enhancing the performance and recovery of Marchador horses and other groups of animals that participate in endurance contests or in frequently repeated exercises.

As previously mentioned, animals trained for endurance competitions tend to have an increased plasma volume, which means there may be some decrease in red blood cell concentration, particularly in erythrocytes (McKeever et al. 1987; McKeever 2002). This adaptation is important to increase the amount of water stored in plasma and reduces the effects of water and mineral loss in horses through hypertonic sweat. Increased plasma volume may also reduce TPP levels. In this study, both groups showed a decrease in TPP levels unrelated to their feed, which was expected, given that their protein levels remained within the range indicated by the NRC (2007) for the type of physical exercise the animals were engaged in. Lastly, it should be kept in mind that Marchador horses may present slight dehydration after riding contests (Wanderley et al. 2010; Melo et al. 2017), and a possible increase in plasma volume may contribute to reducing the negative effects of post-exercise dehydration.

Supplementation with higher calorie density, using a concentrate rich in omega 3 and 6 fatty acids, increases the availability of free fatty acids for the tissues and supports the antioxidant capacity. In this context, an increase in free fatty acids concentration helps to decrease the body temperature and saves glucose during exercise, increasing the time to fatigue. Moreover, increased antioxidant capacity reduces the negative effects of exercise on the body's tissues. Therefore, taken together, these processes support performance and contribute to faster recovery of gaited horses, which often compete several times over the course of a few days in the main horse riding competitions.

In conclusion, the use of diets rich in oil for horses that perform an aerobic exercise could benefit the horses' well-being by increasing the antioxidant capacity and saving glucose levels by utilizing high energy sourced from fat.

\section{Conflict of Interest}

The authors declare that they have no conflicting interests.

\section{Acknowledgements}

The authors would like to thank Guabi Nutrição Animal for providing the feed for the animals; Haras Abreu for providing the animals; and CNPq and CAPES for the granting of scholarships.

\section{References}

Abreu JMG, Manso Filho HC, Manso HECCC 2009: Composição corporal nos cavalo de trabalho. Cien Anim Bras 10: 1122-1127

Barros KV, Carvalho PO, Cassulino AP, Andrade I, West AL, Miles EA, Calder PC, Silveira VL 2013: Fatty acids in plasma, white and red blood cells, and tissues after oral or intravenous administration of fish oil in rats. Clin Nutr 32: 993-998

Coelho C.S, Manso HECCC, Manso Filho HC, Ribeiro Filho JD, Abreu JMG, Escodro PB, Valença SRFA 2018 : Escala para avaliação do bem-estar em equídeos atletas. Rev Bras Med Equina 13: 4-8

Eltweri AM, Thomas AL, Fisk HL, Arshad A, Calder PC, Dennison AR, Bowrey DJ 2017: Plasma and erythrocyte 
uptake of omega-3 fatty acids from an intravenous fish oil based lipid emulsion in patients with advanced oesophagogastric cancer. Clin Nutr 36: 768-774

Ferreira LMC, Melo SKM, Diniz AIA, Vaz SG, Abreu JMG, Manso HECCC, Manso Filho HC 2015: Aerobic exercise produces changes in plasma IL-6 but not IL-1b in four-beat gaited horses, Comp Exe Phys 11: 159-165

Ferreira LMC, Hunka MM, Silva ERR, Melo SKM, Melo ACC, Trindade KLG, Oliveira VTB, Manso HECCC, Manso Filho HC 2017: Hematological and biochemical values in Brazilian four-beat gaited horses, Comp Clin Pathol 26: 321-327

Gleenen SNJ, van Oldruitenborgh-Oosterban MMS, Beynen AC 1999: Dietary fat supplementation and equine plasma lipid metabolism. Eq Vet J 30: 475-478

Hargreaves BJ, Kronfeld DS, Waldron JN, Lopes MA, Gay LS, Saker KE, Cooper W1, Sklan DJ, Harris PA 2002 : Antioxidant status and muscle cell leakage during endurance exercice. Eq Vet J 34: 116-121

Harris P 2009: Feeding management of elite endurance horses. Vet Clin North Am Equine Pract 25: 137-153

Harris PA, Pagan JD, Crandell KG, Davidson N 1999: Effect of feeding thoroughbred horses a high unsaturated or saturated vegetable oil supplemented diet for 6 months following a 10 month fat acclimation. Eq Vet J Suppl 30: $468-474$

Henneke DR, Potter GD, Kreider JL, Yeates BF 1983: Relationship between condition score, physical measurements and body fat percentage in mares. Eq Vet J 15: 371-372

Hollán S 1996: Membrane fluidity of blood cells. Haematologia (Budap) 27: 109-27

Hyyppa S, Saastamoinen M, Pöso AR 1999 Effect of a post exercise fat-supplemented diet on muscle glycogen repletion. Eq Vet J 30: 493-498

Kearns CF, McKeever KH, Abe T 2002: Overview of horse body composition and muscle architecture: implications for performance. Vet J 164: 224-234

Kronfeld DS 1996: Dietary fat affects heat production and other variables of equine performance under hot and humid conditions. Eq Vet J 22: 24-34

Kronfeld DS, Ferrante PL, Grandjean D 1994: Optimal nutrition for athletic performance, with emphasis on fat adaptation in dogs and horses. J Nutr 124: 2745S-2753S

McGreevy P, Berger J, Brauwere N, Doherty O, Harrison A, Fiedler J, Jones C, McDonnell S, McLean A, Nakonechny L, Nicol C, Preshaw L, Thomson P, Tzioumis V, Webster J, Wolfensohn S, Yeates J, Jones B 2018: Using the five domains model to assess the adverse impacts of husbandry, veterinary, and equitation interventions on horse welfare. Animals 8: 41

McKeever KH The endocrine system and the challenge of exercise 2002: Vet Clin N Am Equine Pract 18: 321-353

McKeever KH, Schurg WA, Jarrett SH, Convertino VA 1987: Exercise training-induced hypervolemia in the horses. Med Sci Spo Exe 19: 21-27

Mellor DJ 2017: Operational details of the five domains model and its key applications to the assessment and management of animal welfare. Animals 7: 60

Melo SKM, Ferreira LMC, Hunka MM, Barbosa BL, Abreu JMG, Manso HECCC, Manso Filho HC 2017: Marcha gait simulation test decrease antioxidative biomarkers in four-beat gaited horses. J Eq Vet Sci 55: 12-17

Melo SKM, Vaz SG, Manso ECC, Martins IDS, Hunka MM, Manso HECCC, Manso Filho, HC 2012: Influência da suplementação com concentrado extrusado rico em óleo nos parâmetros hematológicos, biométricos e biomarcadores na digestão de potros. [Influence of supplementation with oil-rich extruded concentrate on hematological and biometric parameters and digestion biomarkers of foals]. Medicina Veterinária (UFRPE) 6: $41-45$

Melo, SKM, Diniz AIA, Lira VL, Muniz SKO, Silva GR, Manso HECCC, Manso Filho HC 2016: Antioxidant and haematological biomarkers in different groups of horses supplemented with polyunsaturated oil and vitamin E. J Ani Phys Ani Nutr 100: 852-859

Moffarts B, Portier K, Kirschvink N, Coudert J, Fellmann N, van Erck E, Letellier C, Motta C, Pincemail J, Art T, Lekeux P 2007: Effects of exercise and oral antioxidant supplementation enriched in (n-3) fatty acids on blood oxidant markers and erythrocyte membrane fluidity in horses. Vet J 174: 113-121

National Research Council - NRC. Nutrient requirements of horses 2007: $6^{\text {th }}$ edn. Washington, D.C. National Academies $360 \mathrm{p}$.

O'Connor CI, Lawrence LM, Hayes SH 2007: Dietary fish oil supplementation affects serum fatty acid concentrations in horses J Anim Sci 85: 2183-2189

Orme CE, Harris RC, Marlin DL, Hurley J 1997: Metabolic adaptation to a fat-supplemented diet by the thoroughbred horse. Br J Nutr 78: 443-458

Wanderley EK, Bem BSC, Melo SKM, Gonzalez JC, Manso HECCC, Manso Filho HC 2015: Hematological and biochemical changes in mangalarga marchador horses after a four-beat gait challenge in three different distances. J Eq Vet Sci 35: 259-263

Wanderley EK, Manso Filho H C, Manso HECCC, Santiago TA, McKeever KH 2010: Metabolic changes in four beat gaited horses after field marcha simulation. Eq Vet J 42: 105-109 